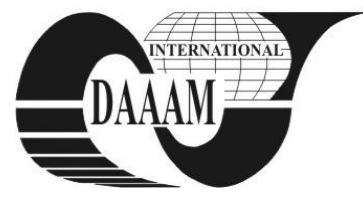

Annals of DAAAM for 2011 \& Proceedings of the 22nd International DAAAM Symposium, Volume 22, No. 1, ISSN 1726-9679 ISBN 978-3-901509-83-4, Editor B. Katalinic, Published by DAAAM International, Vienna, Austria, EU, 2011 Make Harmony between Technology and Nature, and Your Mind will Fly Free as a Bird

\title{
ADAPTIVE MARBLE PLATE CLASSIFICATION SYSTEM BASED ON NEURAL NETWORK AND PLC IMPLEMENTATION
}

\author{
TOPALOVA, I[rina] C[hristova] \& TZOKEV, A[lexander]
}

\begin{abstract}
The process of sorting marble plates according to their surface texture is an important task in the automated marble plate production. Nowadays some inspection systems in marble industry that automates the texture and shade classification tasks are too expensive and are compatible only with specifictechnological equipment in the plant. In this paper a new approach for design of adaptive classification system of marble tiles with similar textures is proposed, aiming at high classification accuracy, applying simple processing and application of only standard devices. It is based on simple image preprocessing, on adaptive training of MLP neural network (MLP NN) with marble histograms and implementation of the algorithm in a Programmable Logic Controller (PLC) for real-time operation. The experimental test results when recognizing marble textures with added motion blur and different illuminations are represented and discussed. The performance of the modeling technique is assessed with different training and test sets. The classification accuracy results are presented and analyzed.
\end{abstract}

Key words: automated classification, neural network, PLC, texture, machine vision

\section{INTRODUCTION}

Many modern systems for automated vision control in stone production industry are developed for inspection and classification of the surface texture of marble slabs and plates after cutting. A major part of them have high market price and are compatible only with the proprietarily company production equipment. The existing software products for texture recognition are not intended for implementation in Programmable Logic Controllers (PLC) widely used for control of technological processes. A specific task in marble plate production is the sorting of tiles with identical textures. The high accuracy classification in this case is difficult because the parametrical descriptions of the textures are high correlated. In addition the textures have to be recognized in movement (during the transportation on the conveyer belt) and with some changes in the illumination of the working area, according to the production process specifics. This is the reason to develop effective methods and algorithms aiming high recognition accuracy for different kinds of similar textures when evaluating them in real production environment conditions. On the other hand optimal (considering accuracy, quick-operating and cost) software and hardware system solutions have to be sought suitable for implementation in PLCs, because these devices are preferable and widely used in up-to-date automated production systems. In this paper a new approach for design of an adaptive classification system of marble plates(ACSMP) with similar textures is developed, aiming high classification accuracy however by simple processing and application of only standard devices.

\section{DESIGN OF THE ACSMP}

\subsection{Texture parametrical description}

There are many contemporary methods and algorithms for texture analysis and recognition. The application of statistical and filter based approaches (Baraldi \& Parmiggiani, 1995) is based on the fact, that there are many software products, offering an opportunity for easy simulation and testing of the results. Context analysis, using "direct local neighborhood" is also lately applied and it is an alternative of a method based only on filtration (Mallick-Goswami \& Datta, 2000). The main disadvantage of the existing methods is the very high computational complexity and relatively low recognition accuracy. All these methods are not efficient when the textures are very similar and have overlapping parametrical descriptions (Sousa \& Pinto, 2004).

In our approach many histograms of each investigated grey scale texture image, corresponding to different movement speeds of the plate and different illuminations are extracted, sampled and stored for further processing. The histogram values are used for training a MLP neural network to classify the textures.

\subsection{Structure of the designed ACSMP}

The functioning structure of the designed ACSMP is shown in Fig.1. After acquisition of the marble plate images through a high resolution CCD industrial camera, they are transferred using Ethernet communication to a conventional PC. The system is working in two modes - off-line or training and online or classification mode.

Off-line mode - in this mode the adaptive training of the system is performed. Histograms of different texture images with different blur effect and illuminations are sampled and arranged in an executable form for adaptive training the MLP neural network. The holdout cross-validation method was applied to estimate the expected level of fit of a model to a data set that is independent of the data that were used to train the model. MLPs with different number of hidden layers, different number of neurons in the input and hidden layers, different transfer functions and different MSE (Mean Square Error) on the output layer were trained and cross-validated. The whole process is performed on the PC. The trained MLP structure and the weight coefficients matrix are downloaded to the PLC neural network database.

On-line mode - in this mode the current extracted histogram is sampled in the PC and transferred to the PLC through the developed communication software, using Multi Point Interface (MPI). The algorithms for a particular neuro - application in the PLC are calculated more precisely, after an unconditional call by the user program. As next a procedure "FDL"-Final Decision Logic for automated evaluation and finding the MLP output with maximal value (corresponding to the recognized texture class) was designed, using ladder diagram method in the PLC-S7-317. The MLP outputs together with FDL results are 
applied to the physical PLC outputs for direct control of the sorting devices.

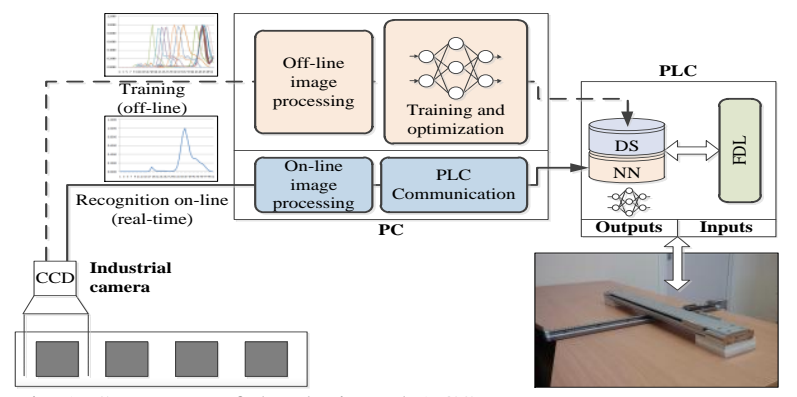

Fig.1. Structure of the designed ACSMP

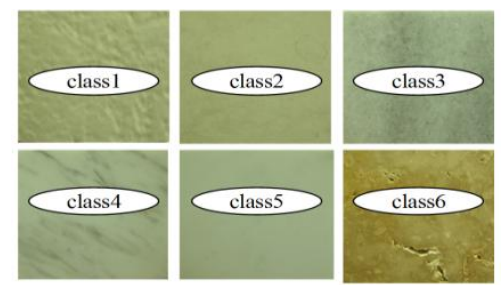

Fig.2. Exemplars of the tested marble textures

\section{EXPERIMENTS AND RESULTS}

The experiments were carried out with the six classes of marble textures shown in Fig.2. The image acquisition was realized with a CCD camera Basler scA1000-20gmwith exposure time of $120 \mu$ s to $29.5 \mathrm{~ms}$ and resolution of 1034 x 779 pixels. The value of 9Pix Motion Blur (MB) corresponds to an image resolution of $300 \mathrm{dpi}$ or $118 \mathrm{Pix} / \mathrm{cm}$, to $25 \mathrm{~m} / \mathrm{min}$ linear velocity of the conveyer belt and to $1 / 500 \mathrm{sec}$ camera exposure time. The MLP structure was trained with sampled histogram values (each $5^{\text {th }}$ value was sampled) with different amount of Motion Blur (3, 6 and 9 Pix MB) and different illuminations Fig.3/a. Standard deviation values (Fig.3/b) for each histogram point $x_{i j}$ related to the mean point value $\overline{x_{i j}}$ for each class $j$ also were calculated as

$$
\sigma_{i j}=\sqrt{\frac{\sum_{i=1}^{N}\left(x_{i j}-\overline{x_{i j}}\right)^{2}}{N-1}}
$$

where $j=$ class number and $i=$ sampled histogram point. The experiments showed that the best fitting of the MLP neural network is obtained when $\sigma$ is between 0.4 and 0.5 (Topalova \& Tzokev, 2010). The optimized MLP was trained with histograms sampling each $5^{\text {th }}$ point and has a three layered structure of 32-64-6. Each of the six classes was trained with 30 exemplars with different MB (max value of 25Pix) and illuminations after 34000 iterations and MSE of 0.07.

a/

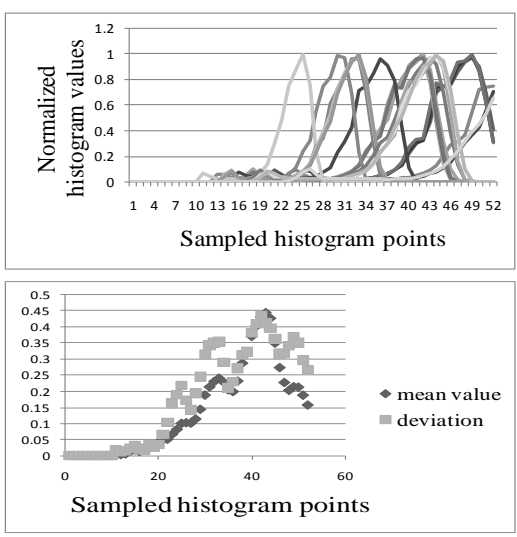

Fig. 3 a/ Training set - sampled histogram points for class 3 with different amount of Motion Blur and different illuminations; $b$ / Standard deviation values for each histogram point

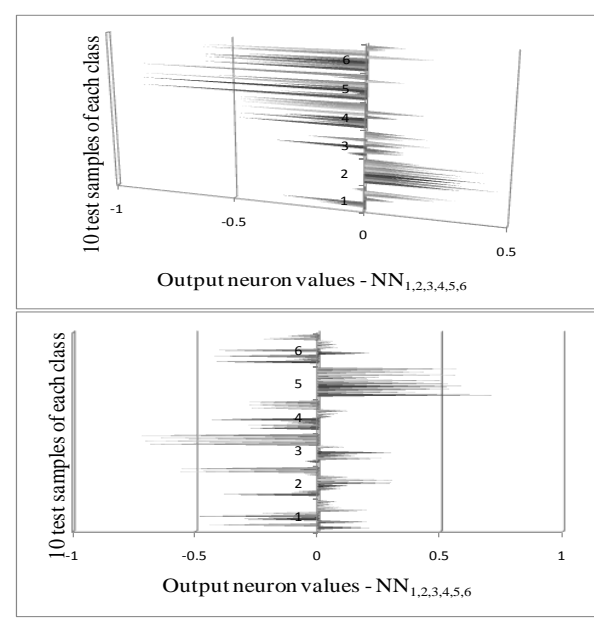

a/

b/

Fig.4. a/ Output neuron values for classification of 10 exemplars of class 2 ; $b$ / Output neuron values for classification of 10 exemplars of class5

The MLP was implemented in PLC SIMATIC S7-317 DP (http://libnodave.sourceforge.net.,2011). The obtained classification accuracy, calculated as the overall number of correct classifications, divided by the number of tested instances (100 exemplars) in the dataset for the six classes is between $87 \%$ and $96 \%$. The maximal value of the time needed for image acquisition, histogram calculation, giving the values to the PLC NN Function Block, recognition through the trained $\mathrm{NN}$ and giving the result to the physical PLC outputs is $578 \mathrm{~ms}$ on the average. The limitations of this research concern the number of tested instances for each of the classes, the number of investigated classes and the PLC platform.

\section{CONCLUSION}

An ACSMP using adaptive classification method was developed and tested with various texture images with added $\mathrm{MB}$ and illuminations to simulate the real production conditions. The developed method shows high recognition accuracy even for motion blur of $25 \mathrm{Pix}$ related to $25 \mathrm{~m} / \mathrm{min}$ linear velocity of the conveyer belt. The proposed adaptive algorithm is implemented and proved for real-time work on standard PLC SIMATIC S7-317 DP instead on FPGA, because PLCs are widely used as control devices in automated production. Thus there is no need to develop additional equipment, adhering to the PLCs. The achieved short maximal system reaction time and the estimated high recognition accuracy is good precondition for proper system work even for higher conveyer belt velocities.

\section{REFERENCES}

Baraldi, A. \& Parmiggiani, F. (1995). An investigation of the Textural Characteristics Associated with Gray Level Cooccurrence Matrix Statistical Parameters, IEEE Trans. OnGeoscienceandRemoteSensing, ISSN:0196-2892, vol. 33, no. 2, pp. 293-304

Mallick, B. \& Datta, A. (2000). Detecting defects in fabric with laser-based morphological image processing, Textile Research Journal, ISSN: 0040-5175, 70: pp.758-762

Topalova, I. \& Tzokev, A. (2010). Automated Classification of Marble Plate Textures with MLP-PLC Implementation, International Conference on Engineering and MetaEngineering: ICEME'2010, ISBN: 978-1-934272-83-1 pp.115-119, 6-9 April, Orlando, USA

Sousa, C. \& Pinto, C. (2004). Comparison of Intelligent Techniques Applied to Marble Classification, ICIAR 2004, ISSN 0302-9743, pp. 802-809, Porto, Portugal

*** (2011) http://libnodave.sourceforge.net/- LIBNODAVE exchange data with Siemens, Accessed on: 2011-1-31 